\title{
Large, isolated, congenital aneurysm of the anterior descending coronary artery
}

\author{
Ying-Tsung Chen, Chung-Lee Hwang, Mee-Nin Kan
}

\begin{abstract}
A large congenital aneurysm, arising from the first diagonal branch of the left anterior descending artery in a 52 year old woman was diagnosed by transoesophageal echocardiography, computed tomography, and magnetic resonance imaging. Surgical closure of the aneurysm was successful.
\end{abstract}

(Br Heart f 1993;70:274-275)

Large congenital aneurysms of the coronary arteries are rare. ${ }^{1-5}$ Most of the reported aneurysms were diagnosed at necropsy, and

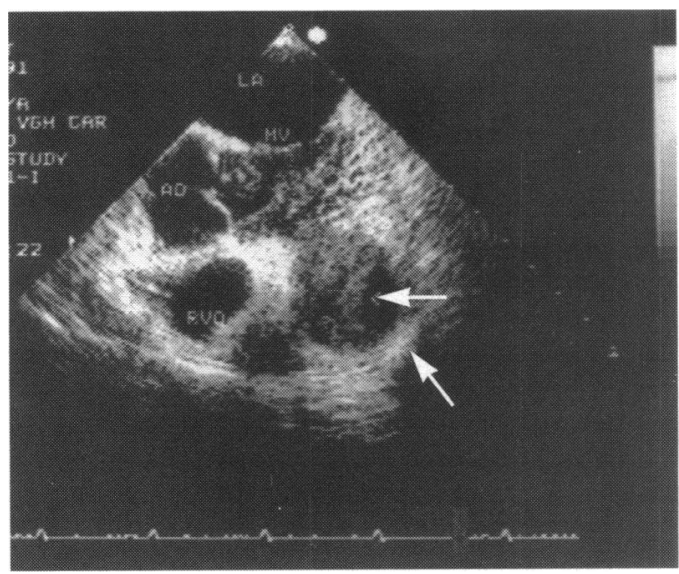

Figure 1 Transoesophageal echocardiogram. Four chamber view showing a round mass (lower arrow) compressing the left ventricle, with thrombus formation (arrow).

Division of

Cardiology, Taichung

Veterans General

Hospital, and National

Yang-Ming Medical

College Triservice

General Hospital,

Chung-Shan Dental

and Medical College,

China Medical

College, Taiwan,

Republic of China

Y-T Chen

C-L Hwang

M-N Kan

Correspondence to

Dr Ying-Tsung Chen,

Division of Cardiology,

Taichung Veterans General

Hospital, 160 Chung-Kang

Road Section 3, Taichung,

Republic of China.
Figure 2 Computed tomogram of the chest showing a large coronary aneurysm arising from the left anterio descending artery, with thrombus formation (arrow). rarely by non-invasive methods before coronary angiography. ${ }^{6} \mathrm{We}$ report a patient in whom a large coronary arterial aneurysm was diagnosed by transoeosophageal echocardiogram, computed tomography, and magnetic resonance imaging before operation.

\section{Case report}

A 52 year old woman was admitted to hospital with a five month history of chest tightness and cough. She had episodes of non-exertional chest tightness lasting five minutes. This was generally followed by coughing that produced mucoid sputum. She was a nonsmoker. Her family history was not helpful and her vital signs and physical examination results were completely normal. Blood, urine, and stool examinations were all normal, as were serum cholesterol, HDL, LDL, and triglycerides. The resting electrocardiogram showed a non-specific $T$ wave change. The chest $x$ ray showed a round soft mass in the left middle lung field that was suspected to be lung cancer. Bronchoscopy showed no abnormal findings. Sputum cytology was negative. A cross sectional echocardiogram showed a round extracardiac mass compressing the left ventricular wall. Transoesophageal echocardiographic imaging showed a cystic mass with necrotising material in the centre of the cavity compressing the left ventricular wall (fig 1).

Computed tomography of the chest showed an oval mass adjacent to the main pulmonary trunk. This mass was enhanced by intravenous injection of contrast medium (fig 2).

Magnetic resonance imaging of the chest showed a well-defined, round, vascular, intrapericardial mass that contained thrombus and compressed the left ventricle. Cardiac catheterisation of the left and right sides of the heart showed normal pressure and cardiac output. A left ventriculogram was normal and showed no wall motion abnormalities. Coronary angiography showed a normal right coronary artery. Selective injection into the left coronary artery showed a solitary aneurysm arising immediately after the origin of the first diagonal branch (fig 3). The other branches of the coronary artery were all normal. The aneurysm was partially filled by thrombus. The patient underwent marsupialisation of the aneurysm. She has remained symptom free since surgery. 


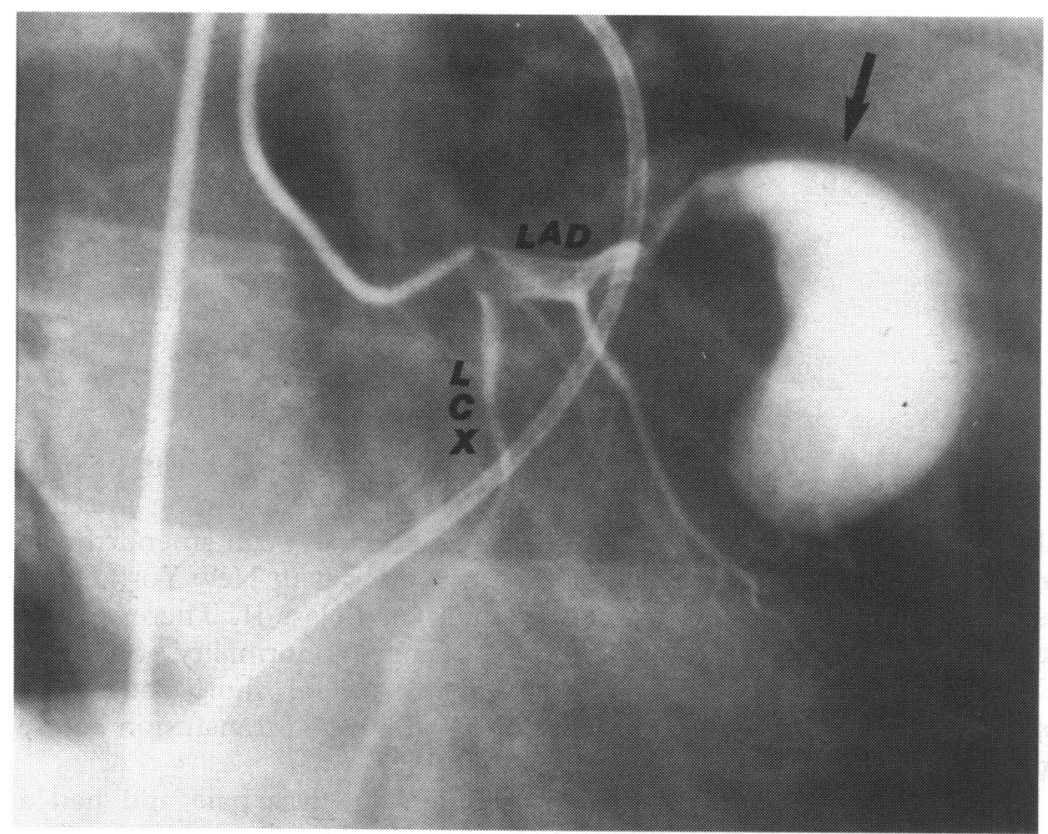

Figure 3 Angiogram of the left coronary artery showing a large aneurysmal sac partially filled by a thrombus formation (arrow).

\section{Discussion}

Aneurysms of the coronary artery are rare and atherosclerosis is the most common cause. Aneurysms can be congenital. The right coronary artery is affected most frequently, followed by the circumflex and left anterior descending coronary arteries. Aneurysms are saccular or fusiform. In our case the aneurysm was probably congenital, because there was no evidence of other possible causes. There were no atherosclerotic lesions in the coronary arteries, and histological studies of the aneurysm did not show important intimal thickening. All of these findings make an atherosclerotic cause very unlikely. Aneurysms of the coronary artery may be asymptomatic and detected incidentally during postmortem examination or coronary angiography. A large aneurysm can be complicated by thrombus formation and embolisation of thrombus into the distal parts of the coronary artery can cause myocardial infarction. Non-invasive techniques, such as echocardiography, computed tomography, and magnetic resonance imaging, can detect aneurysms by their compression of a cardiac chamber. There are too few reports of such cases for a correct diagnosis to be based solely on these non-invasive techniques before coronary angiography. ${ }^{6}$ In our patient the aneurysm appeared as an intrapericardial mass that compressed the left ventricle: it was partially filled by thrombus. This may prove to be a useful feature when non-invasive methods are used to diagnose large coronary aneurysms.

1 Tibbits P, Stanton K, Ashworth H, Baker W: Congenial coronary aneurysms nine years following saphenous vein bypass graft surgery. Ann Thorac Surg 1981;32:411-4.

2 Mattern AL, Baker WP, McHale JJ, Lee DE. Congenital coronary aneurysm with angina pectoris and myocardial infarction treated with saphenous vein bypass graft. $A m$ f Cardiol 1972;30:906-9.

3 Falsetti HL, Carroll RJ. Coronary artery aneurysm: a review of the literature with a report of eleven new cases. Chest 1976;69:630-6.

4 Wilson CS, Weaver WF, Zeman ED, Forker AD. Bilateral nonfistulous congenital coronary arterial aneurysms. $A m$ f Cardiol 1975;35:319-23.

$5 \mathrm{Lim}$ CH, Tan NC, Seah CS, Tan D. Giant congenital aneurysm of the right coronary artery. $\mathrm{Am} \mathcal{f}$ Cardiol 1977;39:751-3.

6 Kenneth GS, Prassad V, Scott AB, Cordie C, Siddesh G Bakr S, Martin AA. Giant coronary artery aneurysm: Detection with echocardiography, computed tomography, and magnetic resonance imaging. Am Heart $f$ phy, and magnetic 